\title{
Long-lasting salt bridges provide the anchoring mechanism of oncogenic Kirsten rat sarcoma proteins at cell membranes
}

\author{
Huixia Lu and Jordi Martí* \\ Department of Physics, Technical University of Catalonia-Barcelona Tech, B4-B5 Northern \\ Campus, Barcelona, Catalonia, Spain \\ E-mail: jordi.marti@upc.edu
}




\begin{abstract}
RAS proteins work as GDP-GTP binary switches and regulate cytoplasmic signalling networks that are able to control several cellular processes, playing an essential role in signal transduction pathways involved in cell growth, differentiation and survival so that overacting RAS signalling can lead to cancer. One of the hardest challenges to face is the design of mutation-selective therapeutic strategies. In this work, a G12D mutated farnesylated GTP bound Kirsten RAt Sarcoma (KRAS) protein has been simulated at the interface of a DOPC/DOPS/cholesterol model anionic cell membrane. A specific long-lasting salt bridge connection between farnesyl and the hypervariable region of the protein has been identified as the main mechanism responsible of the binding of oncogenic farnesylated KRAS-4B to the cell membrane. Free-energy landscapes allowed us to characterise local and global minima of KRAS-4B binding to the cell membrane revealing the main pathways between anchored and released states.
\end{abstract}

\title{
Graphical TOC Entry
}

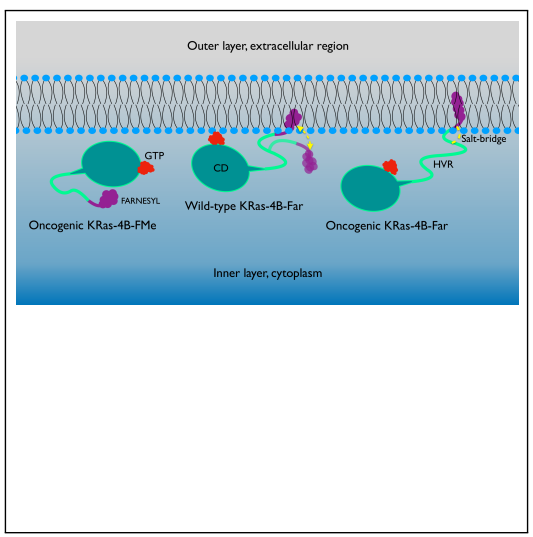

\section{Keywords}

KRAS-4B, oncogenes, anchoring mechanism, model cell membrane, well-tempered metadynamics 
RAS proteins are small guanosine-triphosphatase (GTPase) species that can be activated or de-activated by guanosine-diphosphate (GDP)-guanosine-triphosphate (GTP) switching[1] . Consequently, the interaction of GTP with RAS and their surroundings is an issue of high interest[2]. As surface proteins anchored in the inner leaflet of the cell membrane (i.e. at the cytoplasm), RAS species are normally in the inactive state and they are activated following an incoming signal from their upstream regulators. RAS proteins play an essential role in signal transduction pathways which ultimately turn on genes involved in cell growth, differentiation and survival, eventually leading to cancer[3, 4, 5]. Conventionally, mutant RAS is considered to be defective in GAP-mediated GTP hydrolysis, which results in an accumulation of constitutive GTP-bound RAS in cells. Missense gain-of-function mutations in all three RAS genes are found in $27 \%$ of all human cancers, with $98 \%$ of the mutations at one of three mutational hotspots: G12, G13 and Q61. It has been found that HRAS is the least frequently mutated RAS isoform in human cancers (4\%), whereas KRAS is the predominantly mutated isoform $(85 \%)$, followed by NRAS $(11 \%)$. However, it is unclear how these mutations affect KRAS association with biological membranes[6]. Consequently, we have chosen the specific mutated G12D KRAS-4B as our target oncogenic protein. KRAS has two splice variants, KRAS-4A and KRAS-4B, the latter being found at higher concentration levels[7], in particular in lung, colorectal, and pancreatic cancer cells $[8,9,10,11]$. These insights suggests the importance of fully understanding the regulation of oncogenic KRAS-4B activity when binding on the membrane. KRAS-4B has a highly conserved N-terminal catalytic domain (CD, residues 1-166) and a flexible C-terminal 22-25-amino acid-long, hypervariable region (HVR, residues 167-185)[12]. Apart from different HVR sequences, KRAS-4B is distinguished by its unique HVR post-translational modification (PTM). Usually, HVR lipidation promote RAS anchoring in the plasma membrane. HVR preferentially binds the membrane in the liquid phase and spontaneously inserts its terminal farnesyl (FAR) moiety into the loosely packed phospholipid bilayers[7]. Accumulating evidence indicates that demethylated and farnesylated KRAS-4B (KRAS-4B-Far) could play an important role in the signalling pathways 
that happen on the inner leaflet of the membrane bilayers. KRAS-4B-Far has been reported to be able to be transferred to bind the inner plasma membrane (PM) leaflet. According to Ntai et al. $91 \%$ of the mutant KRAS-4B and $51 \%$ of wild-type KRAS-4B proteins in certain colorectal tumor samples have been found to be of the KRAS-4B-Far isoform[13]. Nevertheless, the effects of KRAS-4B-Far on downstream signalling have yet to be determined. While most efforts have been focused on characterisation of methylated KRAS-4B (KRAS-4B-FMe) binding to the PM, study on KRAS-4B-Far is still an emerging area of research. According to Barcelo et al.[14], phosphorylation at Ser-181 of oncogenic KRAS is required for tumor growth so that in the present work we have chosen to phosphorylate at Ser-181 to model the main mutated oncogenic KRAS-4B protein under study.

In this Letter we shall provide essential information in order to gain precise understanding of the effects of oncogenic mutations on the localisation of wild-type and oncogenic KRAS4B proteins and on their eventual mechanisms to anchor the cell. According to Nussinov et al.[15], the two major pathways in oncogenic RAS-driven proliferation can be promoted when KRAS is membrane-anchored. Furthermore, understanding the structural specifics of KRAS-4B in its GTP-bound form will help to design oncogenic KRAS-4B inhibitors[16]. So, methods of reducing or weakening the interactions between oncogenic KRAS- $4 \mathrm{~B}$ and the membrane with a good knowledge of the structural mechanisms at the atomic level will be a promising target for anticancer drug discovery[17]. On its own, genomics sequence data may not provide the entire information to the oncologist for the selection of targets. Further, the GTP affinity of KRAS-4B is reported to be extremely high, with a dissociation constant of around $10 \mathrm{~mol} / \mathrm{L}$ [18], yet the corresponding binding free-energy hypersurface has been poorly explored. The free-energy landscape (FEL) idea is compelling because it enables us to map many possible conformations which the protein could populate along the different levels of free-energies so that recommendations for treatments might derive from the corresponding FEL, producing further insight into the underlying biological mechanisms and fostering molecular targeting in a significant way[19]. RAS association with membranes 
is not a one-way street and they undergo a cycle of delivery to the PM followed by return to endomembranes for recycling[20]. In this work we have applied well-tempered metadynamics to reveal the affinity of GTP to KRAS-4B and lipids and to obtain the binding free-energy barriers of the process of anchoring of FAR at membrane bilayers from a free-energy perspective, especially for membranes with relatively high contents of cholesterol.

The process of post-translational modifications required to allow KRAS-4B to function properly in cells is represented in Fig. 1: Firstly the prenylation reaction, catalysed by cytosolic farnesyltrasferase (FTase) or geranylgeranyltransferase (GGTase), proceeds through the addition of an isoprenyl group to the Cys-185 side chain. Then farnesylated KRAS-4B is ready for further processing: hydrolysis, catalysed by the endopeptidase enzyme called RAS-converting enzyme 1 (RCE1), during the process the VIM motif (HVR tail composed of three amino-acids: valine-isoleucine-methionine) of the C-terminal Cys-185 is lost in step 2. Later KRAS-4B is transferred to the endoplasmic reticulum for carboxymethylation at the carboxyl terminus of Cys-185 catalysed by isoprenylcysteine carboxyl methyltransferase (ICMT), forming a reversible ester bond. The outcome of these modifications is the farnesylated and methylated KRAS-4B (KRAS-4B-FMe). The reversible ester bond can go through decarboxymethylation, catalysed by prenylated/polyisoprenylated methylated protein methyl esterases (PMPEases) giving rise to a farnesylated and demethylated KRAS-4B (KRAS-4B-Far) which is the product of step 2 and reactant of step 3. This reversible reaction can modulate the equilibrium of methylated/demethylated KRAS-4B population in tumors and consequently can impact downstream signalling, protein-protein interactions, or protein-lipid interactions [21].

After establishing the reliability of the systems studied in the present work using molecular dynamics (MD), very successful to describe a wide variety of molecular setups at the all-atom level, including complex biological systems[22, 23, 24, 25] and metadynamics simulations, able to compute free energy barriers with high precision (see Supporting Information, SI, including a summary table of all simulations carried out), we have analysed the structure 


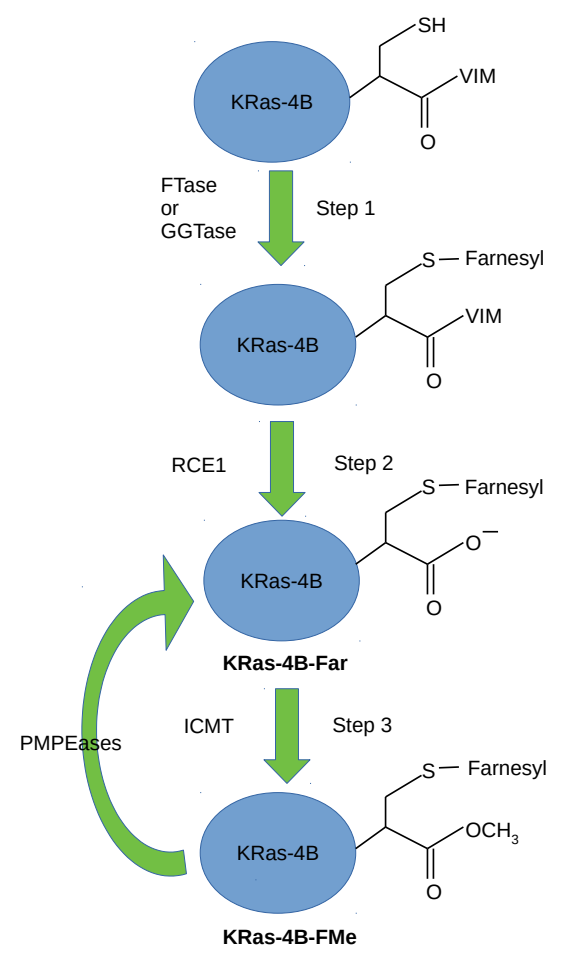

Figure 1: Process of post-translational modifications responsible to the formation of KRAS4B-Far and KRAS-4B-FMe.

of KRAS at the interface of the membrane by means of a series of radial distribution functions (RDF) between selected atomic sites (as described in Figs. S2, S5 and S6). Being the most relevant, RDF of GTP and FAR are shown in Fig. 2. Additional RDF are reported in Fig. S7 (related to CD) and Fig. S8 (related to HVR).

Firstly, hydrogen-bonds (HB) between GTP and active sites of specific aminoacids of the CD have been observed (top of Fig.2). A clear first coordination shell located around 1.75 $\AA$ shall be essentially attributed to HB between selected atom sites. The typical signature of oxygen-hydrogen HB in lipid cell membranes is a maximum located at $1.8 \AA[26]$. For oncogenic KRAS-4B systems, GTP tends to bind the CD of the protein through HB, whereas no HB between GTP and lipids have been observed. From the results reported here, we can observe that GTP prefers binding to the CD of oncogenic KRAS-4B proteins regardless of 

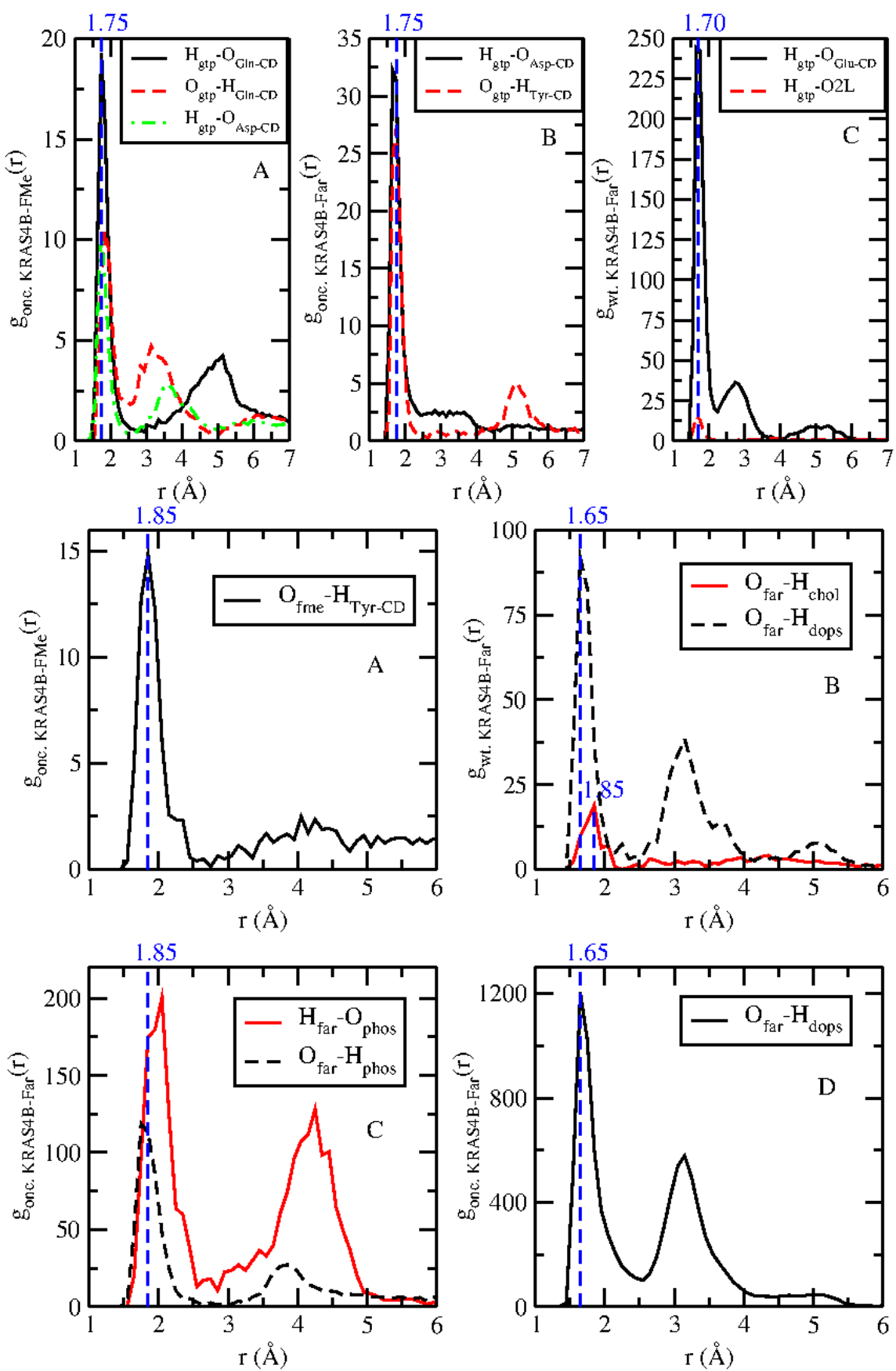

Figure 2: Top three plots: Selected RDF for active atoms of GTP with selected sites of the $\mathrm{CD}$ and active oxygen atoms of head groups of lipids ('O2L'). ' $\mathrm{H}_{g t p}$ ' and ' $\mathrm{O}_{g t p}$ ' represent hydrogen and oxygen atoms from phosphate group of GTP. Bottom four plots: Selected RDF related to selected sites of FAR. Here $\mathrm{O}_{f a r}$ and $\mathrm{H}_{\text {far }}$ correspond to atomic sites belonging to farnesylated KRAS-4B, whereas $\mathrm{O}_{f m e}$ stands for oxygen atoms of methylated KRAS-4B.

carboxymethylation at site Cys-185 before anchoring to the membrane leaflet. In particular, for wild-type KRAS-4B-Far, HB between GTP and the CD are much stronger ( 15-fold) 
than HB between GTP and lipids. However, in the wild-type system GTP is mostly located at the interface of the membrane, close to the CD of KRAS-4B. Especially strong interactions observed between GTP and CD of KRAS-4B-FMe indicate less efficient nucleotide exchange for this mutant. To further analyse the effect of the side chain of CD, calculations were performed revealing that CD is able to associate head groups of lipids, which proves that CD of KRAS-4B plays a role in binding to bilayers in all cases. In the corresponding RDF (Fig. S7), when the two selected oxygen and hydrogen sites are ionised the location of the first shell is usually at distances shorter than $1.8 \AA$. The contribution of the first shell is from a so-called salt bridge[27, 28], which coupling was reported to be of general importance to the stability and function of proteins. A salt bridge has two components: a hydrogen bond and an electrostatic ionic interaction. Very common in proteins are those between the anionic carboxylate $\left(\mathrm{RCOO}^{-}\right)$of aspartic or glutamic acid and the cationic ammonium $\left(\mathrm{RNH}_{3}^{+}\right)$from lysine or the guanidinium $\left(\mathrm{RNHC}\left(\mathrm{NH}_{2}\right)_{2}^{+}\right)$of arginine. Of all non-covalent interactions, salt bridges are among the strongest[29]. In the particular case of KRAS-4B, it has been recently observed that salt bridges can be formed between the protein and PIP2 species[30, 31]. Here we report that the first shell of RDF due to salt bridges between $O_{C D}$ and $H_{\text {dops }}$ is located at $1.65 \AA$ for the three systems (the two oncogenic and the wildtype species of the protein). Nevertheless, it has been shown that HB between the cationic ammonium $\left(\mathrm{RNH}_{3}^{+}\right)$from lysine of $\mathrm{CD}$ of KRAS-4B-FMe and anionic oxygen atoms from DOPS is much stronger than for demethylated KRAS-4B isoforms. Consecutively, RDF related to HVR are displayed in Fig. S8. We have found that HVR is able to form HB and salt bridges with CD, membrane lipids and PHOS. Carboxylmethylation and mutations show their influence on the interactions of HVR with DOPS and PHOS (panels D and F). Whereas the first shell is located at $1.65 \AA$, the salt bridge between $O_{H V R}$ and $H_{\text {dops }}$ is much weaker for oncogenic KRAS-4B-FMe, when comparing with the demethylated case. After phosphorylation (as shown in Fig. S4), oncogenic KRAS-4B proteins (by means of $\mathrm{O}_{\text {phos }}$ ) are able to form stable salt bridges with hydrogen atoms of cationic ammonium from the 
HVR $\left(\mathrm{H}_{H V R}\right)$. However, for wild-type KRAS-4B-Far only weak HB exit between $\mathrm{H}_{H V R}$ and some oxygen atom from the side chain of site Ser-181. As it was pointed out in Refs. [32, 33] our results suggest that CD and HVR have a significant role in binding to the PM, although engaging to the membrane in different ways.

We have investigated meaningful RDF of active sites of FAR with PHOS, CD, and lipids. We obtained (panel D, bottom of Fig.2) that in the oncogenic KRAS-4B-Far system FAR forms strong and long-lasting salt bridges with DOPS, through negatively charged $O_{f a r}$ interacting strongly with the positive charged $H_{\text {dops }}$ of DOPS. In the case of the oncogenic KRAS-4B-FMe, HB are found (panel A) between the carbonyl oxygen of the ester group of Cys-181 $\left(O_{f m e}\right)$, which is weakly basic, and the positive hydrogen of the hydroxyl group of Tyr from $\mathrm{CD}\left(\mathrm{H}_{\text {Tyr-CD }}\right)$. FAR can also interact with cholesterol (panel B) through typical HB and with DOPS through strong salt bridge for wild-type KRAS-4B-Far, which guarantees that the GTP-bound wild-type KRAS-4B-Far could anchor into anionic membrane bilayers. However, the most remarkable fact is seen in panel C (bottom of Fig.2). Only in the oncogenic KRAS-4B-Far long-lasting salt bridges have been located between oxygen and hydrogen atoms from FAR and PHOS, indicating that anchoring of FAR in the oncogenic KRAS4B-Far possesses a large stability provided by the permanent binding of the FAR-PHOS pair. Consequently, the anchoring mechanism described here is playing a key role in order to reach the stability required for oncogenic KRAS proteins to be able to become functional, remaining attached to the inner surface of the plasma membrane through its farnesylated and poylcationic C-terminus[34].

In the snapshot reported in Fig. 3, we described the long-lasting and strong salt bridges existing between active sites of FAR and PHOS, only for the oncogenic KRAS-4B-Far protein.

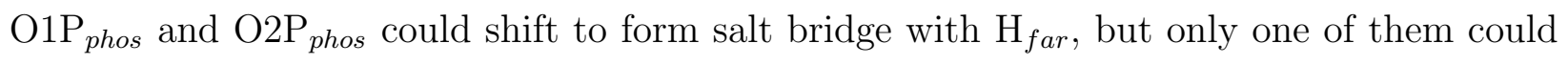
interact with $\mathrm{H}_{f a r}$ hauling the typical $\mathrm{HB}$ distance, with one of them favored by $\mathrm{H}_{\text {far }}$ because of the conformational restrictions in the structure even though both of them share one negative charge. After analysing production trajectories carefully, we have found that $\mathrm{O}_{f a r}$, 


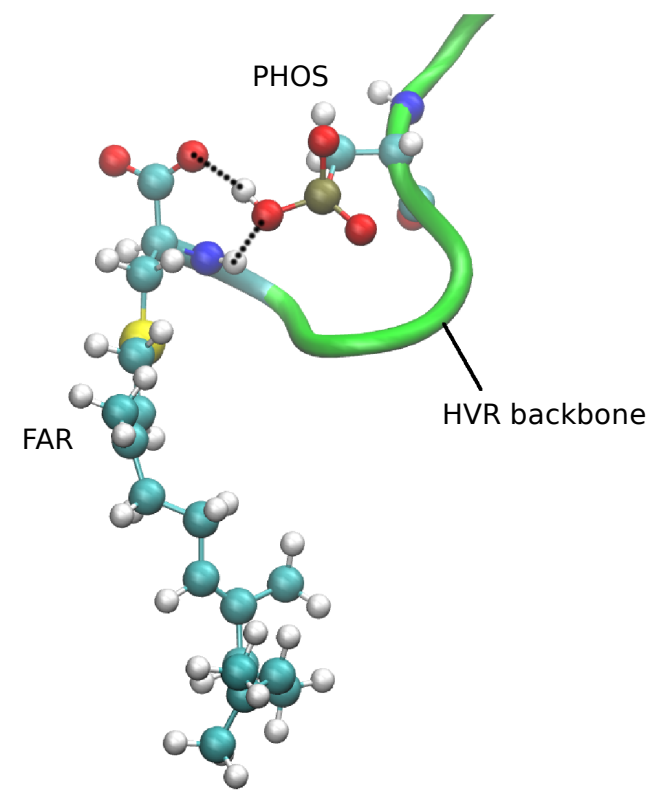

Figure 3: Snapshot of long-lasting salt bridges indicated as black lines of dashes between FAR and PHOS of the oncogenic KRAS-4B-Far. The atoms represented here are: carbon (cyan), oxygen (red), hydrogen (white), nitrogen (blue) and phosphorus (brown). Hydrogen bonds are represented in black dashed lines.

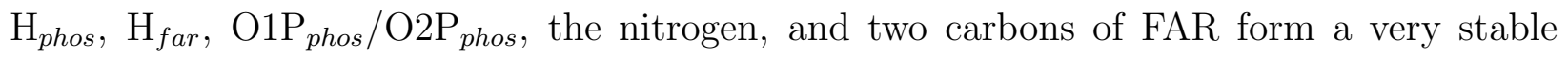
7-membered ring endowing the oncogenic KRAS-4B-Far to get such a specific structure in its tail able to allow the anchoring to PM or reacting with other proteins in vivo. Finally, in order to monitor the interactions between FAR and PHOS for the oncogenic KRAS-4BFar system, we displayed the time evolution of selected atom-atom distances in Fig. S9 regarding the strong salt bridge observed in panel $\mathrm{C}$ (bottom of Fig.2), where we see that the anchoring mechanism described here is fully stable throughout a simulation span of 500 ns. In summary, the finding or design of drugs able to break the long-lasting bonds between FAR and PHOS could be a key factor for the release of oncogenic proteins such as KRAS4B from its anchoring to the membrane towards the internal regions of the cell, eventually becoming harmless.

At the atomic level, the four main configurations corresponding to two stable states of the wild-type KRAS-4B-Far and to the main stable states of oncogenic KRAS-4B-Far and 
KRAS-4B-FMe obtained from MD simulations are illustrated in Fig. 4. We report in Fig. S10 the Z-axis (normal to the XY instantaneous plane of the membrane) positions of center of FAR and GTP from the center of lipids $(\mathrm{z}=0)$ using the second half of the $1000 \mathrm{~ns}$ simulations. After thorough inspection of Fig. S10 and Fig. 4, together with the averaged values reported in Table 1, we report a list of relevant findings in SI.
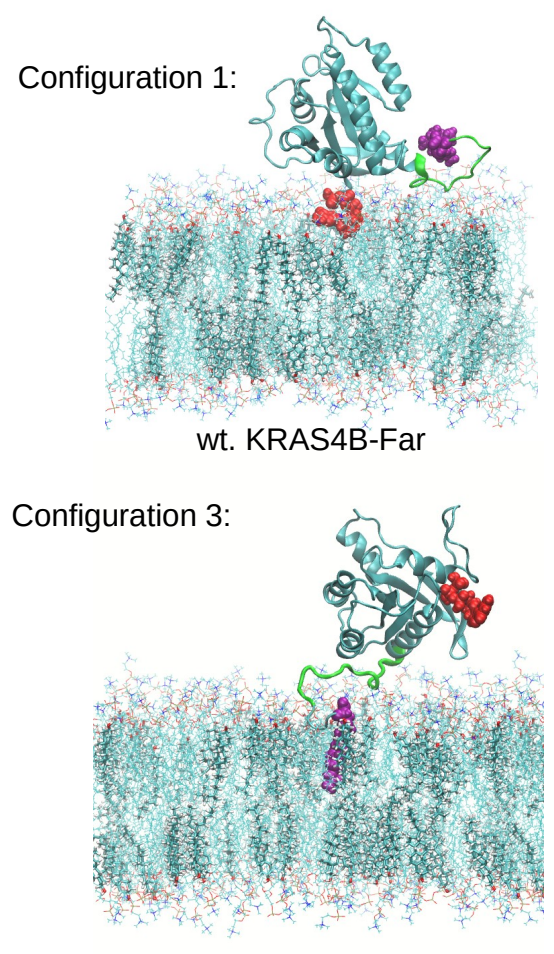

onc. KRAS4B-Far

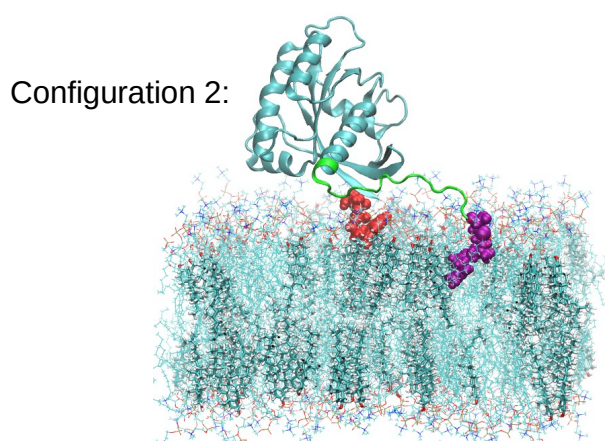

wt. KRAS4B-Far

Configuration 4:

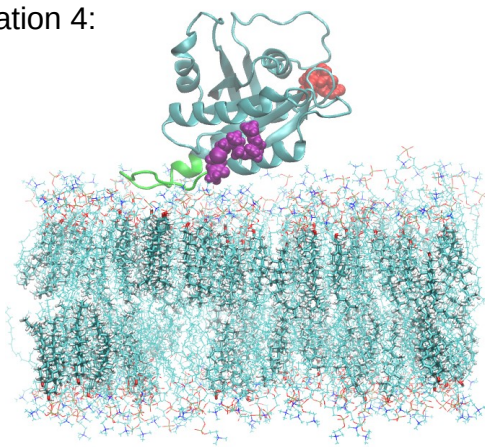

onc. KRAS4B-FMe

Figure 4: Four preferential configurations of the three KRAS-4B-membrane systems obtained from MD simulations at the all-atom level.

Table 1: Averaged values of z-locations (in nm) of FAR, HVR, CD, and GTP for KRAS-4B systems. Values of locations of FAR from configurations 1 and 2 have been provided for the wild-type case. Estimated errors in parenthesis.

\begin{tabular}{ccccc}
\hline Systems & FAR & GTP & HVR & CD \\
\hline wt. KRAS-4B-Far & $3.9(0.48) / 1.73(0.23)$ & $2.40(0.17)$ & $3.93(0.25)$ & $4.48(0.13)$ \\
onc. KRAS-4B-Far & $1.37(0.18)$ & $5.16(0.60)$ & $3.56(0.19)$ & $4.70(0.28)$ \\
onc. KRAS-4B-FMe & $3.82(0.36)$ & $5.07(0.94)$ & $3.84(0.24)$ & $4.70(0.41)$ \\
\hline
\end{tabular}

In order to elucidate the joint effect of phosphorylation and G12D mutation on the 
energetic characteristics of the binding of KRAS-4B in cell membranes, the FEL of wildtype and oncogenic demethylated KRAS-4B-Far proteins have been computed using the well-tempered metadynamics method. A full study of the convergence of the simulations is reported in SI. Transition states between stable minima are not reported, but they could be easily located with the method employed here[35]. Free-energy landscapes are dynamic and conformational states can change in response to intra- and extramolecular events[36, 37], such as the localisations of FAR and GTP. Given the specific interest of the oncogenic and wild-type KRAS-4B-Far proteins in the anchoring of RAS proteins in cell membranes, we have computed two-dimensional (2D) FEL for the corresponding two sets of proteins bound to the anionic membrane composed of DOPC:DOPS:cholesterol (28:7:15) considered above. The hypersurfaces are reported in Fig. 5 and each (meta-)stable state can be indexed by a pair of CV. Several regions with clear minima are present in the two FEL. Since the range of CV space represented in Fig. 5 is rather wide, we will focus especially in the characterisation of free-energy barriers between the particular states of localisations of FAR and GTP in the FEL in the region close to one leaflet of the membrane.

Given that the present 2D FEL include a wide variety of (meta-)stable states, methods able to trace the minimum free-energy path (MFEP) between stable states with high accuracy are highly needed. MFEP can be determined by iteratively refine a pathway connecting stable states that converges to the minimum free-energy trajectories between them. MFEP could also be obtained through the Path Collective Variables description coupled with metadynamics[38]. Alternatively to biased-MD simulation methods, certain spontaneous binding events can also be achieved through extensive standard MD simulations, for example, running for $\mu$ s to ms simulation time[39, 40]. From MFEP we can extract information associated to the most probable trajectories in the $\mathrm{CV}$ space followed by the system involving between (meta-)stable states and it also allows us the determination of local and global transition states. In this way, we have traced the MFEP along the free-energy landscape using the guidelines sketched in SI by means of the R-package metadynminer[41]. Two MFEP have 
been depicted in Fig. 5 where the global minimum is set to zero. The coordinates of minimum free-energy paths are reported in Table S5.
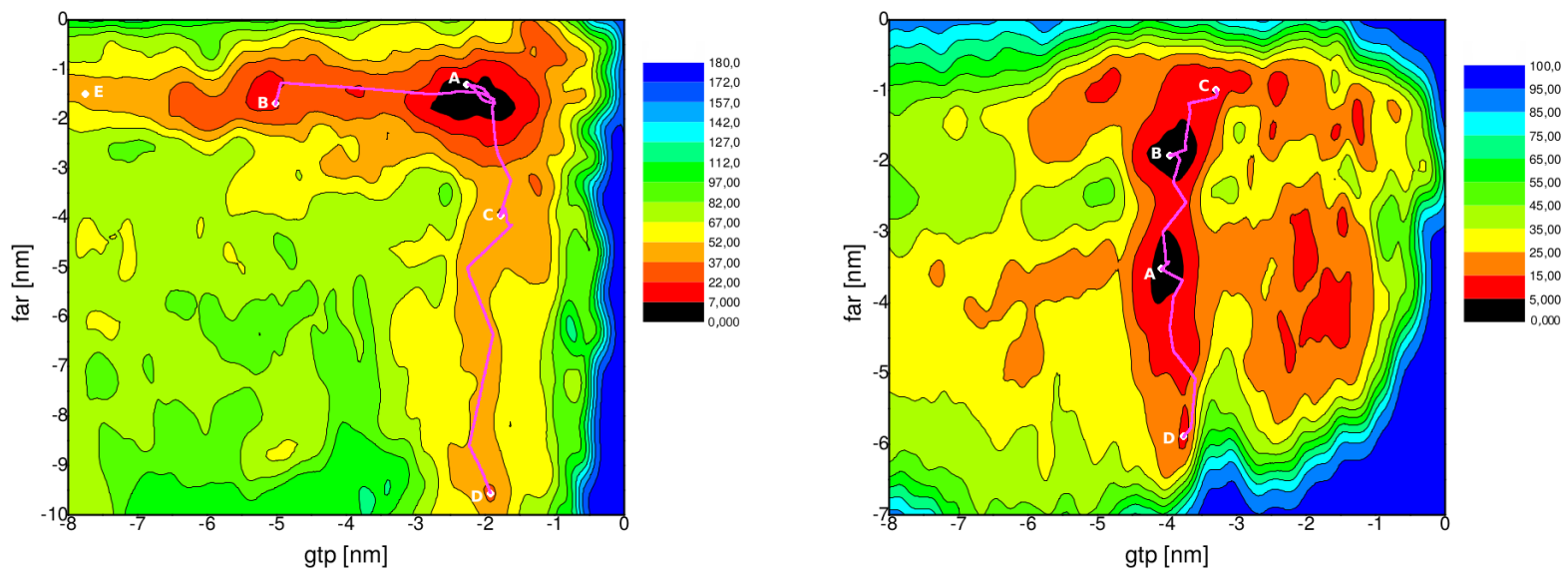

Figure 5: Left: 2D free-energy landscapes $\mathrm{F}$ (gtp, far) (kJ/mol) for the oncogenic KRAS-4BFar system. Several stable and meta-stable configurations (A, B, C, D, E) are indicated. Right: 2D free-energy landscapes $\mathrm{F}$ (gtp, far) $(\mathrm{kJ} / \mathrm{mol}$ ) of wild-type KRAS-4B system. Stable and meta-stable configurations (A, B, C, D) are indicated. All MFEP between two selected basins are depicted in purple and the relevant coordinates have been reported in Table S5.

For oncogenic KRAS-4B-Far protein (left of Fig. 5), the most stable state (global minimum) and four more meta-stable states have been revealed and their coordinates shown in Table S6. The stable or meta-stable configurations that oncogenic KRAS-4B-Far may adopt when bound to the anionic membrane are as follows: in state A, FAR anchors into the "internal" regions of the membrane when GTP is close to the interface and binds the CD moiety as well; in state B, FAR anchors into the "internal" regions of membrane when GTP only binds the CD moiety; in state C FAR has been released off the membrane, but GTP is still bound at the interface; in state D, FAR is solvated by water molecules and GTP only binds the membrane interface and in state E, GTP reaches out further away from the lipidic region and is solvated by the aqueous solution, while FAR keeps being anchored into the membrane. The main findings obtained for the oncogenic case are: (1) While FAR is anchored to the membrane, GTP dissociates from stable state A then binds to the CD moiety (state B), requiring to cross a free-energy barrier of $27.04 \mathrm{~kJ} / \mathrm{mol}$. The final state is 
essentially represented by configuration 3 of Fig. 4; (2) The difference between states C and D is most likely due to the location of HVR. HVR may bind the head groups of lipids (state C) and it can be solvated in the aqueous region (state E). The estimated free-energy barrier $\Delta F$ between states $\mathrm{C}$ and $\mathrm{D}$ is of $17.20 \mathrm{~kJ} / \mathrm{mol}$; (3) We estimated a high free-energy barrier of $42.44 \mathrm{~kJ} / \mathrm{mol}$ from state A to state $\mathrm{C}$, which explains that for oncogenic KRAS-4B-Far, the affinity of FAR to the anionic membrane is extremely high and (4) $\Delta F$ for the transition between states $\mathrm{B}$ and $\mathrm{E}$ has been estimated to be of $27.5 \mathrm{~kJ} / \mathrm{mol}$, corresponding to the energy needed to get the release of GTP from its association from the CD of the protein to water solvation, which explains the high affinity of GTP to CD in the oncogenic KRAS-4B-Far.

Correspondingly, in the case of the FEL of wild-type KRAS-4B-Far protein, two global stable states (A, B) and two more meta-stable states (C, D) have been identified and their coordinates are shown in Table S7 whereas MFEP is shown in Fig.5. In this case the main features are: (1) When FAR is bound to the membrane (state B), a barrier of $10.76 \mathrm{~kJ} / \mathrm{mol}$ should be crossed by FAR shifting to the global stable state A while GTP keeps bound to the CD, but apart from the interface; (2) Shifting from state B to state C (FAR anchored in a deeper position and GTP closer to the interface) can be realised if a free-energy barrier of $6.69 \mathrm{~kJ} / \mathrm{mol}$ is surmounted; (3) When the system shifts its state between A and D (i.e. FAR solvated in the aqueous region), the crossing of a free-energy barrier of $20.10 \mathrm{~kJ} / \mathrm{mol}$ corresponds to the energy needed for the HVR to be released from the anionic membrane and (4) There are several meta-stable states when GTP locates $\sim 2 \mathrm{~nm}$ away from the membrane center, indicating the existence of multiple configurations when GTP is around head groups of lipids, which may play a role in KRAS-4B-Far signal transduction and interactions with other proteins in vivo. As a direct connection to experimental measures[42], it is possible to project the 2D surfaces integration out a single CV. Our numerical results are exhaustively reported in SI (with free-energy profiles reported in Fig.S16).

To summarise the main findings of the work, KRAS-4B belongs to a family of small GTPase that regulate cell growth, differentiation and survival which is frequently mu- 
tated in cancers such as in lung, colon and pancreatic ones. Given that the structural mechanisms at the atomic level of KRAS-4B-membrane association are unknown, we conducted MD simulations of three systems of GTP-bound KRAS-4B (oncogenic KRAS-4BFMe and KRAS-4B-Far, wild-type KRAS-4B-Far) bound to cell membranes constituted by DOPC/DOPS/cholesterol including $\mathrm{KCl}$ solution at $310.15 \mathrm{~K}$ and at the fixed pressure of 1 atm. Each oncogenic KRAS-4B protein contains two mutations: G12D and phosphorylation at its Ser-181 site. KRAS-4B association with the PM requires the penetration of FAR into the membrane and interactions of HVR with lipids in order to allow KRAS-4B a proper localisation in the membrane. We observed that for wild-type KRAS-4B-Far, demethylated Cys-185 allows spontaneous insertion and release of FAR into the anionic membrane bilayer without much difficulty. Conversely, oncogenic KRAS-4B-Far keeps anchored to the membrane and staying in its active site in order to bind upstream regulators and downstream effectors. Further, oncogenic KRAS-4B-FMe stays in an auto-inhibited state in which the HVR is sandwiched between the effector binding site of the CD and the membrane, blocking signal transduction pathways. Interactions such as HB and long-lived salt bridges between different active sites of GTP, the CD, the HVR and lipids have been revealed that to play a central role in the stabilisation of KRAS-4B membrane proteins. In particular, only in the oncogenic KRAS-4B-Far case long-lived salt bridges have been located between oxygen and hydrogen atoms from FAR and PHOS, providing large stability to the anchoring of the KRAS-4B-Far into the PM. We believe this is the key for the permanent association between the oncogenic protein and the inner layer of cell membranes. Further, the information reported in this work could be very valuable to the development of farnesyltransferase inhibitors as anti-cancer agents[43]. Among the two chosen mutations to generate the oncogenic KRAS-4B proteins, we have observed that PHOS has important influence on the behavior of oncogenic KRAS-4B proteins, together with the effect of PTM such as carboxymethylation, which always play a role in the localisation of KRAS-4B at the PM. The reported free-energy profiles of GTP binding to anionic membrane bilayers gave us first-hand information about 
FEL of GTP-bound wild-type/oncogenic KRAS-4B-Far binding to anionic membrane systems. We have located global and local stable states of the two systems that have been chosen to elucidate the joint effect of PHOS and G12D on the KRAS-4B-membrane binding. Our results indicate that for GTP-bound oncogenic KRAS-4B-Far it exists a free-energy barrier of $42.44 \mathrm{~kJ} / \mathrm{mol}$ for the departure of FAR from the PM, which explains the large stability of the FAR moiety of oncogenic KRAS-4B-Far at the anionic membrane. This fully corresponds to the findings reported from MD simulations. Correspondingly, a barrier of $10.76 \mathrm{~kJ} / \mathrm{mol}$ is likely to be crossed for FAR of wild-type KRAS-4B-Far shifting from anchoring to the PM to being solvated by water while GTP keeps bound to the CD (shift between configurations 1 and 2 of Fig. 4). We have estimated a free-energy barrier of $20.10 \mathrm{~kJ} / \mathrm{mol}$ when FAR of the wild-type case shifts from $\sim 4 \mathrm{~nm}$ to $6 \mathrm{~nm}$ away from the membrane center, corresponding to the energies needed for the HVR dissociating from the anionic membrane.

We conducted MD simulations of model anionic (neutral) cell membranes constituted by DOPC (56\%), DOPS (14\%) and cholesterol (30\%) in aqueous ionic solution for the three KRAS-4B isoforms with sequences represented in Fig. S1. The technical details of the simulations are reported in SI. After equilibrating properly the system in the two cases of wild-type/oncogenic KRAS-4B-Far from a $1 \mu$ s run for each system, we switched to run another $1.1 \mu \mathrm{s}$ of well-tempered metadynamics simulations to perform Gibbs free-energy calculations of KRAS-4B binding at anionic phospholipid membrane bilayers, starting from the last configuration of MD simulations. Among the wide variety of methods proposed to obtain free-energy landscapes of multidimensional systems $[2,44,45,46,47,48,49,50$, $51,52]$, we have chosen well-tempered metadynamics, a method able to efficiently explore free-energy surfaces of complex systems using multiple reaction coordinates in complex systems $[53,54,55,56,57]$. Well-tempered metadynamics simulations were performed using the joint GROMACS/2018.3-PLUMED2 tool[58]. Full technical details are reported in SI. Data sets needed to reproduce the two metadynamics simulations reported in the present work can be found in the PLUMED-NEST web page: https://www.plumed-nest.org/eggs/20/028/ 
from the public repository of the PLUMED consortium.

\section{Supporting Information Available}

Full computational details are reported on: (1) system structure and composition; (2) molecular dynamics simulation setups for the full KRAS-4B/DOPC/DOPS/cholesterol membrane system; (3) supplementary results on structure, area per lipid and thickness of the membrane as well as radial distribution functions related to the catalytic domain and hypervariable regions of the protein; (4) well-tempered metadynamics simulations that lead to the construction of the 2D free-energy surfaces as a function of the class of protein, focusing especially on their stability and convergence; (5) technical details about the calculation of minimum free-energy paths. This material is available free of charge via the Internet at http://pubs.acs.org/.

\section{Acknowledgement}

We warmly thank Profs. Carles Calero, Giancarlo Franzese and Dr. Martin Goethe for fruitful discussions. We also thank financial support provided by the Spanish Ministry of Science, Innovation and Universities (project number PGC2018-099277-B-C21, funds MCIU/AEI/FEDER, UE). Dr. Huixia Lu has been a Ph.D. fellow from the Chinese Scholarship Council (grant 201607040059). Computational resources awarded by the Barcelona Supercomputing Center-Spanish Supercomputing Network (grants FI-2018-3-0023, FI-20191-0004, FI-2019-2-0004 and FI-2019-3-0008) are also acknowledged. 


\section{References}

[1] Y. Zhou, P. Prakash, H. Liang, K-J. Cho, A.A. Gorfe, and J.F. Hancock. "Lipidsorting specificity encoded in K-Ras membrane anchor regulates signal output". In: Cell 168.1-2 (2017), pp. 239-251.

[2] A.R. Calixto, C. Moreira, A. Pabis, C. Kötting, K. Gerwert, T. Rudack, and S.C.L. Kamerlin. "GTP hydrolysis without an active site base: A unifying mechanism for ras and related GTPases". In: Journal of the American Chemical Society 141.27 (2019), pp. 10684-10701.

[3] D.S. Goodsell. "The molecular perspective: the ras oncogene". In: Stem cells 17.4 (1999), pp. 235-236.

[4] J. Peli, M. Schröter, C. Rudaz, M. Hahne, C. Meyer, E. Reichmann, and J. Tschopp. "Oncogenic Ras inhibits Fas ligand-mediated apoptosis by downregulating the expression of Fas". In: The EMBO journal 18.7 (1999), pp. 1824-1831.

[5] Y. Pylayeva-Gupta, E. Grabocka, and D. Bar-Sagi. "RAS oncogenes: weaving a tumorigenic web". In: Nature Reviews Cancer 11.11 (2011), pp. 761-774.

[6] M.T. Mazhab-Jafari, C.B. Marshall, M.J. Smith, G.M.C. Gasmi-Seabrook, P.B. Stathopulos, F. Inagaki, L.E. Kay, B.G. Neel, and M. Ikura. "Oncogenic and RASopathyassociated K-RAS mutations relieve membrane-dependent occlusion of the effectorbinding site". In: Proceedings of the National Academy of Sciences 112.21 (2015), pp. $6625-6630$.

[7] H. Jang, S.J. Abraham, T.S. Chavan, B. Hitchinson, L. Khavrutskii, N.I. Tarasova, R. Nussinov, and V. Gaponenko. "Mechanisms of membrane binding of small GTPase KRas4B farnesylated hypervariable region". In: Journal of Biological Chemistry 290.15 (2015), pp. 9465-9477. 
[8] G.A. Hobbs, C.J. Der, and K.L. Rossman. "RAS isoforms and mutations in cancer at a glance". In: Journal of cell science 129.7 (2016), pp. 1287-1292.

[9] I.A. Prior, P.D. Lewis, and C. Mattos. "A comprehensive survey of Ras mutations in cancer". In: Cancer research 72.10 (2012), pp. 2457-2467.

[10] E.D. Pleasance, R.K. Cheetham, P.J. Stephens, D.J. McBride, S.J. Humphray, C.D. Greenman, I. Varela, M.-L. Lin, G.R. Ordóñez, G.R. Bignell, and et al. "A comprehensive catalogue of somatic mutations from a human cancer genome". In: Nature 463.7278 (2010), pp. 191-196.

[11] M. Pek, S.M.J.M. Yatim, Y. Chen, J. Li, M. Gong, X. Jiang, F. Zhang, J. Zheng, X. $\mathrm{Wu}$, and Q. Yu. "Oncogenic KRAS-associated gene signature defines co-targeting of CDK4/6 and MEK as a viable therapeutic strategy in colorectal cancer". In: Oncogene 36.35 (2017), pp. 4975-4986.

[12] K. Koera, K. Nakamura, K. Nakao, J. Miyoshi, K. Toyoshima, T. Hatta, H. Otani, A. Aiba, and M. Katsuki. "K-ras is essential for the development of the mouse embryo". In: Oncogene 15.10 (1997), pp. 1151-1159.

[13] I. Ntai, L. Fornelli, C.J. DeHart, J.E. Hutton, P.F. Doubleday, R.D. LeDuc, A.J. van Nispen, R.T. Fellers, G. Whiteley, E.S. Boja, and et al. "Precise characterization of KRAS4b proteoforms in human colorectal cells and tumors reveals mutation/modification cross-talk". In: Proceedings of the National Academy of Sciences 115.16 (2018), pp. 4140-4145.

[14] C. Barceló, N. Paco, M. Morell, B. Alvarez-Moya, N. Bota-Rabassedas, M. Jaumot, F. Vilardell, G. Capella, and N. Agell. "Phosphorylation at Ser-181 of oncogenic KRAS is required for tumor growth". In: Cancer research 74.4 (2014), pp. 1190-1199.

[15] R. Nussinov, C.-J. Tsai, and H. Jang. "Oncogenic Ras isoforms signaling specificity at the membrane". In: Cancer research 78.3 (2018), pp. 593-602. 
[16] J.M. Ostrem, U. Peters, M.L. Sos, J.A. Wells, and K.M. Shokat. "K-Ras (G12C) inhibitors allosterically control GTP affinity and effector interactions". In: Nature 503.7477 (2013), pp. 548-551.

[17] S. Lu, H. Jang, S. Muratcioglu, A. Gursoy, O. Keskin, R. Nussinov, and J. Zhang. "Ras conformational ensembles, allostery, and signaling". In: Chemical reviews 116.11 (2016), pp. 6607-6665.

[18] P. Liu, Y. Wang, and X. Li. "Targeting the untargetable KRAS in cancer therapy". In: Acta Pharmaceutica Sinica B (2019).

[19] R. Nussinov, H. Jang, C.-J. Tsai, and F. Cheng. "Precision medicine and driver mutations: Computational methods, functional assays and conformational principles for interpreting cancer drivers". In: PLoS computational biology 15.3 (2019), e1006658.

[20] J.T. Swarthout, S. Lobo, L. Farh, M.R. Croke, W.K. Greentree, R.J. Deschenes, and M.E. Linder. "DHHC9 and GCP16 constitute a human protein fatty acyltransferase with specificity for H-and N-Ras". In: Journal of Biological Chemistry 280.35 (2005), pp. 31141-31148.

[21] H. Abdelkarim, A. Banerjee, P. Grudzien, N. Leschinsky, M. Abushaer, and V. Gaponenko. "The Hypervariable Region of K-Ras4B Governs Molecular Recognition and Function". In: International Journal of Molecular Sciences 20.22 (2019), p. 5718.

[22] M. Karplus and G.A. Petsko. "Molecular dynamics simulations in biology". In: Nature 347.6294 (1990), pp. 631-639.

[23] M. Karplus and J.A. McCammon. "Molecular dynamics simulations of biomolecules". In: Nature structural biology 9.9 (2002), pp. 646-652.

[24] G. Nagy, M.C. Gordillo, E. Guàrdia, and J. Marti. "Liquid water confined in carbon nanochannels at high temperatures". In: The Journal of Physical Chemistry B 111.43 (2007), pp. 12524-12530. 
[25] P.E. Videla, J. Sala, J. Marti, E. Guàrdia, and D. Laria. "Aqueous electrolytes confined within functionalized silica nanopores". In: The Journal of chemical physics 135.10 (2011), p. 104503.

[26] H. Liu, H. Zhang, and B. Jin. "Fluorescence of tryptophan in aqueous solution". In: Spectrochimica Acta Part A: Molecular and Biomolecular Spectroscopy 106 (2013), pp. 54-59.

[27] Z.S. Hendsch and B. Tidor. "Do salt bridges stabilize proteins? A continuum electrostatic analysis". In: Protein Science 3.2 (1994), pp. 211-226.

[28] B.H. Honig and W.L. Hubbell. "Stability of" salt bridges" in membrane proteins". In: Proceedings of the National Academy of Sciences 81.17 (1984), pp. 5412-5416.

[29] E.V. Anslyn and D.A. Dougherty. Modern physical organic chemistry. University science books, 2006.

[30] M.C. Gregory, M.A. McLean, and S.G. Sligar. "Interaction of KRas4b with anionic membranes: A special role for PIP2". In: Biochemical and biophysical research communications 487.2 (2017), pp. 351-355.

[31] S. Cao, S. Chung, SJ. Kim, Z. Li, D. Manor, and M. Buck. "K-Ras G-domain binding with signaling lipid phosphatidylinositol $(4,5)$-phosphate (PIP2): membrane association, protein orientation, and function". In: Journal of Biological Chemistry 294.17 (2019), pp. 7068-7084.

[32] D. Abankwa, A.A. Gorfe, K. Inder, and J.F. Hancock. "Ras membrane orientation and nanodomain localization generate isoform diversity". In: Proceedings of the National Academy of Sciences 107.3 (2010), pp. 1130-1135.

[33] K. Weise, S. Kapoor, C. Denter, J. Nikolaus, N. Opitz, S. Koch, G. Triola, A. Herrmann, H. Waldmann, and R. Winter. "Membrane-mediated induction and sorting of K-Ras microdomain signaling platforms". In: Journal of the American Chemical Society 133.4 (2011), pp. 880-887. 
[34] L. Janosi and A.A. Gorfe. "Segregation of negatively charged phospholipids by the polycationic and farnesylated membrane anchor of Kras". In: Biophysical journal 99.11 (2010), pp. 3666-3674.

[35] H. Lu and J. Marti. "Cellular absorption of small molecules: free energy landscapes of melatonin binding at phospholipid membranes". In: Scientific Reports 10.1 (2020), pp. $1-12$.

[36] S. Kumar, B. Ma, C-J. Tsai, N. Sinha, and R. Nussinov. "Folding and binding cascades: dynamic landscapes and population shifts". In: Protein science 9.1 (2000), pp. 10-19.

[37] K. Gunasekaran, B. Ma, and R. Nussinov. "Is allostery an intrinsic property of all dynamic proteins?" In: Proteins: Structure, Function, and Bioinformatics 57.3 (2004), pp. $433-443$.

[38] M. Bernetti, M. Masetti, M. Recanatini, R.E. Amaro, and A. Cavalli. "An Integrated Markov State Model and Path Metadynamics Approach To Characterize Drug Binding Processes". In: Journal of chemical theory and computation 15.10 (2019), pp. 56895702.

[39] I. Buch, T. Giorgino, and G. De Fabritiis. "Complete reconstruction of an enzymeinhibitor binding process by molecular dynamics simulations". In: Proceedings of the National Academy of Sciences 108.25 (2011), pp. 10184-10189.

[40] Y. Shan, E.T. Kim, M.P. Eastwood, R.O. Dror, M.A. Seeliger, and D.E. Shaw. "How does a drug molecule find its target binding site?" In: Journal of the American Chemical Society 133.24 (2011), pp. 9181-9183.

[41] P. Hošek and V. Spiwok. "Metadyn View: Fast web-based viewer of free energy surfaces calculated by metadynamics". In: Computer Physics Communications 198 (2016), pp. 222-229. 
[42] J.P.M. Jambeck and A.P. Lyubartsev. "Exploring the free energy landscape of solutes embedded in lipid bilayers". In: The Journal of Physical Chemistry Letters 4.11 (2013), pp. $1781-1787$.

[43] A. Kazi, S. Xiang, H. Yang, L. Chen, P. Kennedy, M. Ayaz, S. Fletcher, C. Cummings, H. R Lawrence, F. Beato, and et al. "Dual farnesyl and geranylgeranyl transferase inhibitor thwarts mutant KRAS-driven patient-derived pancreatic tumors". In: Clinical Cancer Research 25.19 (2019), pp. 5984-5996.

[44] M. Mezei. "Adaptive umbrella sampling: Self-consistent determination of the nonBoltzmann bias". In: Journal of Computational Physics 68.1 (1987), pp. 237-248.

[45] C. Bartels and M. Karplus. "Multidimensional adaptive umbrella sampling: Applications to main chain and side chain peptide conformations". In: Journal of Computational Chemistry 18.12 (1997), pp. 1450-1462.

[46] F.S. Csajka, J. Marti, and D. Chandler. "Finding reaction pathways of complex systems." In: Abstracts of Papers of The American Chemical Society. Vol. 213. 1997, 99PHYS.

[47] J. Marti, F.S. Csajka, and D. Chandler. "Stochastic transition pathways in the aqueous sodium chloride dissociation process". In: Chem. Phys. Lett. 328.1 (2000), pp. 169-176.

[48] C. Dellago, P.G. Bolhuis, and P.L. Geissler. "Transition path sampling". In: Advances in Chemical Physics 123 (2002), pp. 1-78.

[49] J. Marti and F.S. Csajka. "Transition path sampling study of flip-flop transitions in model lipid bilayer membranes". In: Physical Review E 69.6 (2004), p. 061918.

[50] D. Laria, J. Marti, and E. Guardia. "Protons in supercritical water: a multistate empirical valence bond study". In: Journal of the American Chemical Society 126.7 (2004), pp. 2125-2134. 
[51] C. Dellago and P.G. Bolhuis. "Transition path sampling and other advanced simulation techniques for rare events". In: Advanced Computer Simulation Approaches for Soft Matter Sciences III. Springer, 2009, pp. 167-233.

[52] J. Henin, G. Fiorin, C. Chipot, and M.L. Klein. "Exploring multidimensional free energy landscapes using time-dependent biases on collective variables". In: Journal of Chemical Theory and Computation 6.1 (2009), pp. 35-47.

[53] G. Bussi, F.L. Gervasio, A. Laio, and M. Parrinello. "Free-energy landscape for $\beta$ hairpin folding from combined parallel tempering and metadynamics". In: Journal of the American Chemical Society 128.41 (2006), pp. 13435-13441.

[54] M. Deighan, M. Bonomi, and J. Pfaendtner. "Efficient simulation of explicitly solvated proteins in the well-tempered ensemble". In: Journal of Chemical Theory and Computation 8.7 (2012), pp. 2189-2192.

[55] J.C. Palmer, R. Car, and P.G. Debenedetti. "The liquid-liquid transition in supercooled ST2 water: a comparison between umbrella sampling and well-tempered metadynamics". In: Faraday Discussions 167 (2013), pp. 77-94.

[56] S. Haldar, P. Kührová, P. Banáš, V. Spiwok, J. Sponer, P. Hobza, and M. Otyepka. "Insights into stability and folding of GNRA and UNCG tetraloops revealed by microsecond molecular dynamics and well-tempered metadynamics". In: Journal of Chemical Theory and Computation 11.8 (2015), pp. 3866-3877.

[57] J. Marti. "Free-energy surfaces of ionic adsorption in cholesterol-free and cholesterolrich phospholipid membranes". In: Molecular Simulation 44.13-14 (2018), pp. 11361146.

[58] G.A. Tribello, M. Bonomi, D. Branduardi, C. Camilloni, and G. Bussi. "PLUMED 2: New feathers for an old bird". In: Computer Physics Communications 185.2 (2014), pp. 604-613. 